\title{
Deutsche Bildungslandschaft fordert Umdenken beim ZDF
}

http://doi.org/10.1515/bd-2020-0019

„Bildung hat kein Ablaufdatum.” Unter dieser Prämisse fordern die Bildungsgewerkschaft GEW, der Deutsche Bibliotheksverband e.V. und Wikimedia Deutschland e.V. den ZDF-Fernsehrat heute in einem gemeinsamen offenen Brief ${ }^{1}$ auf, die dauerhafte Veröffentlichung öffentlich-rechtlicher Bildungssendungen und -inhalte zu garantieren. Der Sender will nach aktuellen Plänen Bildungsinhalte nach fünf Jahren aus dem Netz nehmen - und beraubt damit unter anderem Schulen, Bibliotheken und freie Wissensressourcen wie Wikipedia der Möglichkeit der langfristigen Nutzung.

„Lehrerinnen und Lehrer greifen auf Dokumentationen zurück, wenn sie gut sind, nicht nur, weil sie neu sind. Die Wissenschaft und freie Wissenssammlungen wie Wikipedia setzen auf Belege, die dauerhaft online sind. Bibliotheken bieten kostenfreien Zugang zu Wissen und Informationen. Ihre Nutzerinnen und Nutzer erwarten, dass Inhaltsangebote in Bibliotheken auf Dauer und nicht zeitlich begrenzt zur Verfügung stehen”, erklären die Bildungsträger in ihrem heute publizierten Schreiben. Zusammen rufen sie das ZDF auf, Bildungsinhalte nicht zwangsweise aus dem Netz zu löschen.

„Nur Beiträge, die dauerhaft veröffentlicht sind, können zuverlässig in der deutschsprachigen Wikipedia oder in anderen Bildungsmaterialien verlinkt werden. Für die Freiwilligen, die Artikel in der deutschsprachigen Wikipedia aktuell halten, wie für die täglich neun Millionen Nutzerinnen und Nutzer wäre die Verfügbarkeit von Bildungsinhalten wie Dokumentationen, Erklärvideos und Grafiken des ZDF ohne Verfallsdatum von enormem Vorteil”, sagt Abraham Taherivand von Wikimedia Deutschland.

„Bibliotheken stellen ihren Nutzerinnen und Nutzern auch digitale Inhalte zur Verfügung, die in der Schule, Universität und für die Weiterbildung benötigt werden. Dies gehört zum Informations- und Bildungsauftrag öffentlicher und wissenschaftlicher Bibliotheken“, so Prof. Dr. Andreas Degkwitz, Bundesvorsitzender des Deutschen Bibliotheksverbandes e.V. „Dabei gehen Bibliotheken wie ihre Nutzerinnen und Nutzer selbstverständlich von einer dauerhaften Zugänglichkeit der zur Verfügung gestellten Inhalte aus.“

1 https://www.bibliotheksverband.de/fileadmin/user_upload/DBV/positionen/Offener_Brief_ dbv_GEW_wikimedia.pdf.pdf [Zugriff: 21.11.2019]. 
Die Nachfrage nach guten Inhalten besteht unabhängig davon, wann die Inhalte hochgeladen wurden. Das zeigen die Abrufzahlen, die das ZDF für den Fall „Terra X“ selbst in seinem Änderungskonzept ausführt: 40 \% der Sichtungen entfielen 2018 auf Videos, die in den Vorjahren veröffentlicht wurden. „Wenn ich gute Bilder habe, baue ich die natürlich in meinen Unterricht ein. Nehme ich eine Reportage oder eine Doku, die in den Vorjahren gelaufen ist, bricht mein Konzept bald wieder zusammen. Das ist doch haarsträubend”, so Dr. Ilka Hoffmann aus dem GEW-Vorstand.

\section{Bildungsauftrag der Öffentlich-Rechtlichen}

Die ARD hat mit ihrer Themenwoche Bildung viele Ansätze für das digitale Lernen beleuchtet und Expertinnen und Experten Gehör verschafft. Dass der öffentlichrechtliche Rundfunk selbst qua Auftrag Teil der deutschen Bildungslandschaft ist, wird gerne vergessen.

\section{Der ZDF-Fernsehrat}

Der Fernsehrat besteht aus Mitgliedern unterschiedlicher gesellschaftlicher Gruppen, er soll die Interessen der Zuschauerinnen und Zuschauer gegenüber dem ZDF vertreten. Zu seinen Aufgaben gehört es, das Programm des ZDF zu kontrollieren und den Intendanten zu beraten, den der Fernsehrat für die Dauer von fünf Jahren wählt. Der Fernsehrat tagte am 13. Dezember 2019 in Magdeburg.

\section{Ansprechpartner:}

Deutscher Bibliotheksverband e.V. (dbv)

Barbara Schleihagen, Bundesgeschäftsführerin

Tel.: 030/644 98 99-25, E-Mail: schleihagen@bibliotheksverband.de

\section{Gewerkschaft Erziehung und Wissenschaft (GEW)}

Dr. Ilka Hoffmann, Mitglied des Geschäftsführenden Vorstands, OB Schule Tel.: 069/78973-324, E-Mail: ilka.hoffmann@gew.de

\section{Wikimedia Deutschland}

Abraham Taherivand, Geschäftsführender Vorstand

Tel.: 030/219 158 26-0, E-Mail: abraham.taherivand@wikimedia.de 